PROCEEDINGS OF THE

AMERICAN MATHEMATICAL SOCIETY

Volume 127, Number 9, Pages 2591-2600

S 0002-9939(99)05037-6

Article electronically published on April 9, 1999

\title{
IDENTITIES OF INCOMPLETE KLOOSTERMAN SUMS
}

\author{
YE YANGBO
}

(Communicated by Dennis A. Hejhal)

\begin{abstract}
Identities between incomplete Kloosterman sums and incomplete hyper-Kloosterman sums are established.
\end{abstract}

\section{INTRODUCTION}

In recent years various identities of Kloosterman sums and other exponential sums have been proved and their roles in relative trace formulas and Langlands' functoriality conjecture have been studied (Jacquet and Ye [3], Mao and Rallis [6], Ye [7], [8], [9], [10], and Zagier [11]). Some of these identities imply the fundamental lemmas of relative trace formulas and hence provide us new information about the corresponding functoriality of group representations.

More specifically, an identity is proved in Ye [10] between a complete hyperKloosterman sum and an exponential sum over a quadratic number field. The fundamental lemma of a relative trace formula for $G L_{n}$ would follow from this identity and other conjectured identities. This relative trace formula would in turn characterize quadratic base change for $G L_{n}$.

In Ye [9] an identity is established between an exponential sum of Kloosterman type and an exponential sum over a cyclic algebraic number field of a prime degree. Although we do not know whether this identity is related to a relative trace formula, it provides us a new interpretation of Davenport-Hasse relations of Gauss sums (Davenport and Hasse [2]).

On the other hand Kloosterman sums and related exponential sums have important applications in number theory, based on estimation of individual sums and their weighted sums. We are especially interested in estimation of incomplete Kloosterman sums and incomplete hyper-Kloosterman sums. In Bump, Duke, Hoffstein, and Iwaniec [1] estimation of hyper-Kloosterman sums was used in improving bounds of Fourier coefficients of Maass cusp forms. Moreover, Luo, Rudnick, and Sarnak in [5] established a new lower bound of the first eigenvalue for Maass cusp forms based in part on estimates of hyper-Kloosterman sums. Recently Luo in [4] established a new bound for incomplete hyper-Kloosterman sums.

In this article we will establish identities between incomplete hyper-Kloosterman sums and incomplete classical Kloosterman sums in the case of prime power moduli. We will see that these incomplete sums are essentially the same and hence estimation of one sum implies estimation of the other sum.

Received by the editors November 18, 1997.

1991 Mathematics Subject Classification. Primary 11L05; Secondary 11F70.

The author was supported in part by NSF Grant \#DMS 97-01225. 
From the viewpoint of base change this identity is closely related to the identity in Ye [9]. Indeed, we may regard a local hyper-Kloosterman sum as the lifting of a local Kloosterman sum to the direct sum of $n$ copies of local field $\mathbb{Q}_{p}$. Then the exponential sum in [9] is the lifting of an exponential sum of Kloosterman type to an unramified extension field of $\mathbb{Q}_{p}$ of degree $n$.

Our first result is an identity between incomplete exponential sums. We denote $\mathrm{e}(x)=e^{2 \pi i x}$ and set $\varepsilon_{p}=1$ if $p \equiv 1(\bmod 4)$ and $\varepsilon_{p}=i$ if $p \equiv 3(\bmod 4)$. We will use Legendre symbols.

Theorem 1. Let $c>1$ be an odd integer such that $p^{2} \mid c$ for any prime factor $p$ of c. Let $e$ be a nonzero integer and $m>1$ an integer satisfying $m \equiv 1(\bmod \phi(c))$, where $\phi$ is the Euler function. For any $z$ with $(z, c)=1$ and any $x$ with $1 \leq x \leq c$ we have

$$
\begin{aligned}
& \sum_{\substack{1 \leq b_{1} \leq x, \bmod c, b_{2}, \ldots, b_{m} \\
\left(b_{1}, c\right)=\cdots=\left(b_{m}, c\right)=1}} \mathrm{e}\left(\frac{1}{c}\left(b_{1}+\cdots+b_{m}+z b_{1}^{e} \bar{b}_{2} \cdots \bar{b}_{m}\right)\right) \\
& =c^{(m-1) / 2}\left(\prod_{\substack{p \mid c \text { to an } \\
\text { odd power }}}\left(\frac{c_{2} \cdots c_{r}}{p}\right) \varepsilon_{p}^{r}\right) \sum_{\substack{1 \leq b \leq x,(b, c)=1}} \mathrm{e}\left(\frac{1}{c}\left(b+z b^{e}(\overline{2-m})\right)\right)
\end{aligned}
$$

where $y \bar{y} \equiv 1(\bmod c)$ for any $y$ with $(y, c)=1$. Here for each prime $p$ which divides $c$ to an odd power the even constant $r$ with $1<r \leq m-3$ and the nonzero integers $c_{2}, \ldots, c_{r}$ can be solely determined by $p$ and $m$.

For instance, when $p=3$, we have $r=(m-1) / 3$ and $c_{2}=\cdots=c_{r}=1$. For the general case see the proof of Lemma 1 . If we choose $e=\phi(c)-1$ in Theorem 1 , we can get an identity between incomplete Kloosterman sums and incomplete hyper-Kloosterman sums.

Theorem 2. Let $c>1$ be an odd integer such that $p^{2} \mid c$ for any prime factor $p$ of c. Let $m>1$ be an integer satisfying $m \equiv 1(\bmod \phi(c))$. For any $z$ with $(z, c)=1$ and any $x$ with $1 \leq x \leq c$ we have

$$
\begin{aligned}
& \sum_{\substack{1 \leq b_{1} \leq x, b_{2}, \ldots, b_{m} \\
\left(b_{1}, c\right)=\cdots=\left(b_{m}, c\right)=1}} \mathrm{e}\left(\frac{1}{c}\left(b_{1}+\cdots+b_{m}+z \bar{b}_{1} \cdots \bar{b}_{m}\right)\right) \\
& =c^{(m-1) / 2}\left(\prod_{\substack{p \mid c \text { to an } \\
\text { odd power }}}\left(\frac{c_{2} \cdots c_{r}}{p}\right) \varepsilon_{p}^{r}\right) \sum_{\substack{1 \leq b \leq x,(b, c)=1}} \mathrm{e}\left(\frac{1}{c}(b+z(\overline{2-m}) \bar{b})\right) .
\end{aligned}
$$

\section{MELlin tRAnsforms}

2.1. Mellin transforms of hyper-Kloosterman sums. Following the methods used in Ye [9] we can deduce an identity between hyper-Kloosterman sum and the classical Kloosterman sum. Moreover, this identity can be written in terms of incomplete exponential sums. Let $p$ be an odd prime and $q=p^{a}$ with $a \geq 1$. Fix a nonzero integer $e$ and an additive character $\psi_{p}$ of order zero of the local field $\mathbb{Q}_{p}$. Denote by $R_{p}$ the ring of integers in $\mathbb{Q}_{p}$ and by $R_{p}^{\times}$the group of invertible elements 
of $R_{p}$. Let $S$ be a subset of $R_{p}^{\times}$such that $S=S\left(1+q R_{p}\right)$. For $m>1$ we define an incomplete exponential sum

$$
K l_{m}(z ; e ; S)=\sum_{\substack{b_{1} \in S /\left(1+q R_{p}\right), b_{2}, \ldots, b_{m} \in R_{p}^{\times} /\left(1+q R_{p}\right)}} \psi_{p}\left(\frac{1}{q}\left(b_{1}+\cdots+b_{m}+\frac{z b_{1}^{e}}{b_{2} \cdots b_{m}}\right)\right)
$$

where $z \in R_{p}^{\times}$. If we take $e=-1$, then $K l_{m}(z ; e ; S)$ becomes an incomplete hyperKloosterman sum. Let $\chi$ be any multiplicative character of $\mathbb{Q}_{p}$. Then the Mellin transform of this incomplete exponential sum is

$$
\begin{aligned}
& \int_{R_{p}^{\times}} \chi^{-1}(z) K l_{m}(z ; e ; S) d z \\
& \quad=q^{m} \int_{\substack{z \in R_{p}^{\times}, b_{1} \in S, b_{2}, \ldots, b_{m} \in R_{p}^{\times}}} \chi^{-1}(z) \psi_{p}\left(\frac{1}{q}\left(b_{1}+\cdots+b_{m}+\frac{z b_{1}^{e}}{b_{2} \cdots b_{m}}\right)\right) d z d b_{1} \cdots d b_{m} .
\end{aligned}
$$

Changing variables from $z$ to $x=z b_{1}^{e} /\left(b_{2} \cdots b_{m} q\right)$ and from $b_{i}$ to $y_{i}=b_{i} / q$ for $i=1, \ldots, m$, we get

$$
\begin{aligned}
& \int_{R_{p}^{\times}} \chi^{-1}(z) K l_{m}(z ; e ; S) d z \\
& (1) \quad=q^{-1} \chi^{-1}\left(q^{m-e}\right) \\
& \quad \cdot \int_{\substack{y_{1} \in q^{-1} S, x, y_{2}, \ldots, y_{m} \in q^{-1} R_{p}^{\times}}} \chi^{-1}\left(x y_{1}^{-e} y_{2} \cdots y_{m}\right) \psi_{p}\left(x+y_{1}+\cdots+y_{m}\right) d x d y_{1} \cdots d y_{m} .
\end{aligned}
$$

In this section we will assume that $a>1$ and consider three cases: (i) $\chi$ is ramified with its conductor exponent $a(\chi)=a$, (ii) $\chi$ is ramified with $a(\chi) \neq a$, and (iii) $\chi$ is unramified.

2.2. The case of $a(\chi)=a>1$. In this case the integral $\int_{q^{-1} R_{p}^{\times}} \chi^{-1}(x) \psi_{p}(x) d x$ equals the local $\varepsilon$-factor $\varepsilon\left(\chi, \psi_{p}\right)$. Therefore

$$
\begin{aligned}
& \int_{R_{p}^{\times}} \chi^{-1}(z) K l_{m}(z ; e ; S) d z \\
& \quad=q^{-1} \chi^{-1}\left(q^{m-e}\right)\left(\varepsilon\left(\chi, \psi_{p}\right)\right)^{m} \int_{q^{-1} S} \chi^{-1}\left(y_{1}^{-e}\right) \psi_{p}\left(y_{1}\right) d y_{1} .
\end{aligned}
$$

We need a lemma to evaluate $\varepsilon\left(\chi, \psi_{p}\right)^{m}$ :

Lemma 1. Let $p$ be an odd prime satisfying $(p, m)=(p-1, m)=1$ and $m \equiv$ $1(\bmod \phi(q))$. Let $\chi$ be a ramified character with the conductor exponent $a(\chi)=$ 
$a>1$. Set $q=p^{a}$. Then

$$
\begin{aligned}
& \left(\varepsilon\left(\chi, \psi_{p} ; d x\right)\right)^{m-1} \\
& =q^{(m-1) / 2} \chi\left(\frac{q^{m-1}}{2-m}\right) \quad \text { if a is even; } \\
& =q^{(m+r-1) / 2} \chi\left(\frac{q^{m-1}}{2-m}\right) \\
& \cdot \int_{z_{1}, \ldots, z_{r} \in p^{(a-1) / 2} R_{p}} \chi\left(1+z_{1}^{2}+c_{2} z_{2}^{2}+\cdots+c_{r} z_{r}^{2}\right) d z_{1} \cdots d z_{r}
\end{aligned}
$$

if $a$ is odd

where $1<r \leq m-3$ and $\left(c_{2}, p\right)=\cdots=\left(c_{r}, p\right)=1$. Here $r \equiv 0(\bmod 2)$, and $c_{2}, \ldots, c_{r} \in \mathbf{Z}$ are solely determined by $p$ and $m$.

Proof. We start from computation of $\varepsilon\left(\chi, \psi_{p} ; d x\right)^{m}$ :

$$
\begin{aligned}
&\left(\varepsilon\left(\chi, \psi_{p} ; d x\right)\right)^{m}= \int_{\left(q^{-1} R_{p}^{\times}\right)^{m}} \chi^{-1}\left(x_{1} \cdots x_{m}\right) \psi_{p}\left(x_{1}+\cdots+x_{m}\right) d x_{1} \cdots d x_{m} \\
&= q^{m} \chi\left(q^{m}\right) \int_{\left(R_{p}^{\times}\right)^{m}} \chi^{-1}\left(y_{1}^{m} y_{2} \cdots y_{m}\right) \\
& \cdot \psi_{p}\left(\frac{y_{1}\left(1+y_{2}+\cdots+y_{m}\right)}{q}\right) d y_{1} \cdots d y_{m}
\end{aligned}
$$

where we changed variables from $x_{1}, \ldots, x_{m}$ to $y_{1}=q x_{1}, y_{2}=q x_{2} / y_{1}, \ldots, y_{m}=$ $q x_{m} / y_{1}$. Since $m \equiv 1(\bmod \phi(q))$ and the conductor exponent of $\chi$ is $a$, we have $\chi\left(y_{1}^{m}\right)=\chi\left(y_{1}\right)$. Consequently

$$
\begin{aligned}
\left(\varepsilon\left(\chi, \psi_{p} ; d x\right)\right)^{m}=q^{m-1} \chi\left(q^{m-1}\right) & \int_{\substack{x \in q^{-1} R_{p}^{\times}, y_{2}, \ldots, y_{m} \in R_{p}^{\times}}} \chi^{-1}\left(x y_{2} \cdots y_{m}\right) \\
& \cdot \psi_{p}\left(x\left(1+y_{2}+\cdots+y_{m}\right)\right) d x d y_{2} \cdots d y_{m}
\end{aligned}
$$

where $x=y_{1} / q$. From $a(\chi)=a>1$ we conclude that the integral with respect to $x$ is non-zero only when $1+y_{2}+\cdots+y_{m} \in R_{p}^{\times}$. Therefore

$$
\begin{aligned}
\left(\varepsilon\left(\chi, \psi_{p} ; d x\right)\right)^{m} & =q^{m-1} \chi\left(q^{m-1}\right) \varepsilon\left(\chi, \psi_{p} ; d x\right) \\
\cdot & \int_{\substack{y_{2}, \ldots, y_{m} \in R_{p}^{\times}, 1+y_{2}+\cdots+y_{m} \in R_{p}^{\times}}} \chi\left(\frac{1+y_{2}+\cdots+y_{m}}{y_{2} \cdots y_{m}}\right) d y_{2} \cdots d y_{m} .
\end{aligned}
$$

To compute the integral with respect to $y_{2}, \ldots, y_{m}$ we set $y_{2}=y(1+u)$ with $u \in p^{[(a+1) / 2]} R_{p}$ and $y \in R_{p}^{\times} /\left(1+p^{[(a+1) / 2]} R_{p}\right)$ with $y \notin-\left(1+y_{3}+\cdots+y_{m}\right)+p R_{p}$. Then the integral with respect to $y_{2}$ becomes a sum with respect to $y$ of integrals 
with respect to $u$ :

$$
\begin{aligned}
\sum_{y} \int_{p^{[(a+1) / 2]} R_{p}} & \chi\left(\frac{1+y(1+u)+y_{3}+\cdots+y_{m}}{y y_{3} \cdots y_{m}(1+u)}\right) d u \\
= & \sum_{y} \chi\left(\frac{1+y+y_{3}+\cdots+y_{m}}{y y_{3} \cdots y_{m}}\right) \\
& \cdot \int_{p^{[(a+1) / 2]} R_{p}} \chi\left(1-\frac{1+y_{3}+\cdots+y_{m}}{1+y+y_{3}+\cdots+y_{m}} u\right) d u
\end{aligned}
$$

because any higher powers of $u$ are contained in the conductor of $\chi$. We observe that the integrand on the right side is an additive character of $u$; hence the integral with respect to $u$ is non-zero only if $1+y_{3}+\cdots+y_{m} \in p^{[a / 2]} R_{p}$. Note here $[a / 2] \geq 1$ because $a>1$. Therefore the integral on the right side of (2) is actually taken over $y_{2}, \ldots, y_{m} \in R_{p}^{\times}$with $1+y_{3}+\cdots+y_{m} \in p^{[a / 2]} R_{p}$. By similar arguments we conclude that for this integral the variables $y_{2}, \ldots, y_{m} \in R_{p}^{\times}$satisfy conditions

$$
\begin{aligned}
1+y_{3}+\cdots+y_{m} & \in p^{[a / 2]} R_{p}, \\
1+y_{2}+y_{4}+\cdots+y_{m} & \in p^{[a / 2]} R_{p}, \\
\cdots \cdots \cdots & \\
1+y_{2}+\cdots+y_{m-1} & \in p^{[a / 2]} R_{p} .
\end{aligned}
$$

From these conditions we can see that $y_{3}, \ldots, y_{m} \in y_{2}+p^{[a / 2]} R_{p}$. Then the condition $1+y_{3}+\cdots+y_{m} \in p^{[a / 2]} R_{p}$ further implies that $1+(m-2) y_{2} \in p^{[a / 2]} R_{p}$, i.e., $y_{2}, \ldots, y_{m} \in-1 /(m-2)+p^{[a / 2]} R_{p}$. Setting $y_{i}=-\left(1+x_{i}\right) /(m-2)$ with $x_{i} \in p^{[a / 2]} R_{p}$ for $i=2, \ldots, m$, we can write the integral on the right side of (2) as

$$
\begin{gathered}
\int_{\left(p^{[a / 2]} R_{p}\right)^{m-1}} \chi\left((2-m)^{m-2} \frac{1+x_{2}+\cdots+x_{m}}{\left(1+x_{2}\right) \cdots\left(1+x_{m}\right)}\right) d x_{2} \ldots d x_{m} \\
\quad=\chi\left((2-m)^{m-2}\right) \int_{\left(p^{[a / 2]} R_{p}\right)^{m-1}} \chi\left(1-\sum_{2 \leq i<j \leq m} x_{i} x_{j}\right) d x_{2} \ldots d x_{m} .
\end{gathered}
$$

Since $m \equiv 1(\bmod \phi(q))$, we can replace $\chi\left((2-m)^{m-2}\right)$ by $\chi^{-1}(2-m)$. When $a>1$ is even, this last integral equals

$$
\begin{gathered}
\int_{\substack{y_{2}, \ldots, y_{m} \in R_{p}^{\times}, 1+y_{2}+\cdots+y_{m} \in R_{p}^{\times}}} \chi\left(\frac{1+y_{2}+\cdots+y_{m}}{y_{2} \cdots y_{m}}\right) d y_{2} \cdots d y_{m} \\
=q^{(1-m) / 2} \chi^{-1}(2-m) .
\end{gathered}
$$

When $a>1$ is odd, the integrand on the right side of equation (3) becomes $\chi\left(1-x_{2}\left(x_{3}+\cdots+x_{m}\right)-\sum_{2<i<j \leq m} x_{i} x_{j}\right)$, and hence the integral with respect to $x_{2}$ is nonzero only if $x_{3}+\cdots+x_{m} \in p^{(a+1) / 2} R_{p}$. When this is the case the integral with respect to $x_{2}$ equals $p^{-(a-1) / 2}$. We then can set $x_{3}=-\left(x_{4}+\cdots+x_{m}\right)+p^{(a+1) / 2} R_{p}$. 
By integrating with respect to $x_{3}$ we get

$$
\begin{aligned}
& \int_{\substack{y_{2}, \ldots, y_{m} \in R_{p}^{\times} \\
1+y_{2}+\cdots+y_{m} \in R_{p}^{\times}}} \chi^{-1}\left(\frac{1+y_{2}+\cdots+y_{m}}{y_{2} \cdots y_{m}}\right) d y_{2} \cdots d y_{m} \\
& =p^{-a} \chi^{-1}(2-m) \\
& \quad \cdot \int_{x_{4}, \ldots, x_{m} \in p^{(a-1) / 2} R_{p}} \chi\left(1+\left(x_{4}+\cdots+x_{m}\right)^{2}-\sum_{3<i<j \leq m} x_{i} x_{j}\right) d x_{4} \cdots d x_{m} .
\end{aligned}
$$

To complete the square for $x_{4}$ we set $z_{1}=x_{4}+\left(x_{5}+\cdots+x_{m}\right) / 2 \in p^{(a-1) / 2} R_{p}$; the integrand then becomes

$$
\chi\left(1+z_{1}^{2}+\frac{3}{4}\left(x_{5}+\cdots+x_{m}\right)^{2}-\sum_{4<i<j \leq m} x_{i} x_{j}\right) .
$$

If $p \neq 3$ we can complete the square for $x_{5}$. If $p=3$, then the integrand simplifies to

$$
\chi\left(1+z_{1}^{2}-\sum_{4<i<j \leq m} x_{i} x_{j}\right)
$$

and we can repeat the above computation to integrals with respect to $x_{5}, \ldots, x_{m}$. By an induction argument we conclude that

$$
\begin{aligned}
& \int_{\substack{y_{2}, \ldots, y_{m} \in R_{p}^{\times}, 1+y_{2}+\cdots+y_{m} \in R_{p}^{\times}}} \chi^{-1}\left(\frac{1+y_{2}+\cdots+y_{m}}{y_{2} \cdots y_{m}}\right) d y_{2} \cdots d y_{m} \\
& =q^{(r-m+1) / 2} \chi^{-1}(2-m) \\
& \quad \cdot \int_{z_{1}, \ldots, z_{r} \in p^{(a-1) / 2} R_{p}} \chi\left(1+z_{1}^{2}+c_{2} z_{2}^{2}+\cdots+c_{r} z_{r}^{2}\right) d z_{1} \cdots d z_{r}
\end{aligned}
$$

when $a>1$ is odd, where $1<r \leq m-3, r \equiv m-1(\bmod 2)$, and $c_{2}, \ldots, c_{r} \in R_{p}^{\times}$. Since $m \equiv 1(\bmod \phi(q))$, we have $m \equiv 1(\bmod 2)$ and $r \equiv 0(\bmod 2)$. By changing variables we can further assume that $c_{2}, \ldots, c_{r}$ are nonzero integers relatively prime to $p$. The results from (2), (4), and (5) prove the Lemma.

When $a>1$ is even, Lemma 1 implies that

$$
\begin{aligned}
\int_{R_{p}^{\times}} \chi^{-1}(z) K l_{m}(z ; e ; S) d z & \\
\quad=q^{(m-3) / 2} \chi^{-1}\left(q^{1-e}(2-m)\right) \varepsilon\left(\chi, \psi_{p}\right) & \int_{q^{-1} S} \chi^{-1}\left(y^{-e}\right) \psi_{p}(y) d y .
\end{aligned}
$$


Since $a(\chi)=a$ the above can be written as

$$
\begin{gathered}
q^{(m-3) / 2} \chi^{-1}\left(q^{1-e}(2-m)\right) \int_{q^{-1} R_{p}^{\times}} \chi^{-1}(x) \psi_{p}(x) d x \int_{q^{-1} S} \chi^{-1}\left(y^{-e}\right) \psi_{p}(y) d y \\
=q^{(m-1) / 2} \chi^{-1}(2-m) \int_{R_{p}^{\times}} \chi^{-1}(z) d z \sum_{y \in S /\left(1+q R_{p}\right)} \psi_{p}\left(\frac{y+z y^{e}}{q}\right)
\end{gathered}
$$

where we set $z=q^{1-e} x y^{-e}$ and then changed variables from $y$ to $y / q$. If we change variables from $z$ to $x=(2-m) z$ we finally get

$$
\begin{aligned}
& \int_{R_{p}^{\times}} \chi^{-1}(z) K l_{m}(z ; e ; S) d z \\
& \quad=q^{(m-1) / 2} \int_{R_{p}^{\times}} \chi^{-1}(x) d x \sum_{y \in S /\left(1+q R_{p}\right)} \psi_{p}\left(\frac{1}{q}\left(y+\frac{x y^{e}}{2-m}\right)\right) .
\end{aligned}
$$

When $a(\chi)=a>1$ is odd we get that

$$
\begin{aligned}
& \int_{R_{p}^{\times}} \chi^{-1}(z) K l_{m}(z ; e ; S) d z \\
& =q^{(m+r-3) / 2} \chi\left(\frac{q^{e-1}}{2-m}\right) \\
& \text {. } \int_{\left(p^{(a-1) / 2} R_{p}\right)^{r}} \chi\left(1+z_{1}^{2}+c_{2} z_{2}^{2}+\cdots+c_{r} z_{r}^{2}\right) d z_{1} \cdots d z_{r} \\
& \int_{\substack{x \in q^{-1} R_{p}^{\times} \\
y \in q^{-1} S}} \chi^{-1}\left(x y^{-e}\right) \psi_{p}(x+y) d x d y .
\end{aligned}
$$

Changing variables from $y$ to $y / q$ and from $x$ to $z$ by

$$
x=\frac{z y^{e}}{(2-m) q}\left(1+z_{1}^{2}+c_{2} z_{2}^{2}+\cdots+c_{r} z_{r}^{2}\right)
$$

we get

$$
\begin{aligned}
& q^{(m+r+1) / 2} \int_{R_{p}^{\times}} \chi^{-1}(z) d z \int_{S} \psi_{p}\left(\frac{1}{q}\left(y+\frac{z y^{e}}{2-m}\right)\right) d y \\
& \cdot \int_{\left(p^{(a-1) / 2} R_{p}\right)^{r}} \psi_{p}\left(\frac{z y^{e}}{(2-m) q}\left(z_{1}^{2}+c_{2} z_{2}^{2}+\cdots+c_{r} z_{r}^{2}\right)\right) d z_{1} \cdots d z_{r} .
\end{aligned}
$$

Changing variables from $z_{1}, \ldots, z_{r}$ to $x_{i}=z_{i} p^{(1-a) / 2} \in R_{p}$ for $i=1, \ldots, r$, the innermost integral becomes

$$
\begin{array}{r}
p^{r(1-a) / 2} \int_{\left(R_{p}\right)^{r}} \psi_{p}\left(\frac{z y^{e}}{p}\left(z_{1}^{2}+c_{2} z_{2}^{2}+\cdots+c_{r} z_{r}^{2}\right)\right) d z_{1} \cdots d z_{r} \\
=q^{-r / 2} \gamma\left(\frac{2 z y^{e}}{p}, \psi_{p}\right) \gamma\left(\frac{2 c_{2} z y^{e}}{p}, \psi_{p}\right) \cdots \gamma\left(\frac{2 c_{r} z y^{e}}{p}, \psi_{p}\right) .
\end{array}
$$


Here we used the formula

$$
\int_{R_{p}} \psi_{p}\left(\frac{b x^{2}}{2}\right) d x=|b|_{p}^{-1 / 2} \gamma\left(b, \psi_{p}\right)
$$

for $|b|_{p}>1$, where $\gamma\left(b, \psi_{p}\right)$ is the Weil constant.

Now we want to choose a special character $\psi_{p}$. Let $\varphi=\varphi_{\mathbb{R}} \prod_{p} \varphi_{p}$ be a character of the adele ring $\mathbf{A}$ of $\mathbb{Q}$ which is trivial on $\mathbb{Q}$ such that $\varphi_{\mathbb{R}}(x)=e^{2 \pi i x}=\mathrm{e}(x)$ and the order of $\varphi_{p}$ is zero for every prime $p$. Let $c=p_{1}^{a_{1}} \cdots p_{s}^{a_{s}}$ be an odd positive integer where $p_{1}, \ldots, p_{s}$ are distinct primes and every $a_{j}>1$ for $j=1, \ldots, s$. Now we define a character $\psi_{p_{j}}$ for each $j=1, \ldots, s$ by $\psi_{p_{j}}(x)=\bar{\varphi}_{p_{j}}\left(x p_{j}^{a_{j}} / c\right)$ for any $x \in \mathbb{Q}_{p}$. Then the order of each $\psi_{p_{j}}$ is still zero. When $p=p_{j}$ we have

$$
\begin{aligned}
& q^{-r / 2} \gamma\left(\frac{2 z y^{e}}{p}, \psi_{p}\right) \gamma\left(\frac{2 c_{2} z y^{e}}{p}, \psi_{p}\right) \cdots \gamma\left(\frac{2 c_{r} z y^{e}}{p}, \psi_{p}\right) \\
& \quad=q^{-r / 2} \gamma\left(\frac{2 z y^{e} p_{j}^{a_{j}}}{p c}, \bar{\varphi}_{p}\right) \gamma\left(\frac{2 c_{2} z y^{e} p_{j}^{a_{j}}}{p c}, \bar{\varphi}_{p}\right) \cdots \gamma\left(\frac{2 c_{r} z y^{e} p_{j}^{a_{j}}}{p c}, \bar{\varphi}_{p}\right) .
\end{aligned}
$$

Because of $p>2$ and our specific choice of character $\varphi$, we have

$$
\gamma\left(\frac{2 c_{i} z y^{e} p_{j}^{a_{j}}}{p c}, \bar{\varphi}_{p}\right)=\left(\frac{\left.2 c_{i} z y^{e} \overline{\left(c p_{j}^{-a_{j}}\right.}\right)}{p}\right) \varepsilon_{p}
$$

for $i=1, \ldots, r$, where $x \bar{x} \equiv 1(\bmod p)$. Here we use the Legendre symbol, and set $\varepsilon_{p}=1$ if $p \equiv 1(\bmod 4)$ and $\varepsilon_{p}=i$ if $p \equiv 3(\bmod 4)$. Consequently

$$
\begin{aligned}
\int_{R_{p}^{\times}} \chi^{-1}(z) K l_{m}(z ; e ; S) d z \\
=q^{(m+1) / 2} \int_{R_{p}^{\times}} \chi^{-1}(z) d z \int_{S} \psi_{p}\left(\frac{1}{q}\left(y+\frac{z y^{e}}{2-m}\right)\right) d y \\
\quad \cdot\left(\frac{2 z y^{e}\left(\overline{c p_{j}^{-a_{j}}}\right)}{p}\right)\left(\frac{2 c_{2} z y^{e}\left(\overline{c p_{j}^{-a_{j}}}\right)}{p}\right) \cdots\left(\frac{2 c_{r} z y^{e}\left(\overline{c p_{j}^{-a_{j}}}\right)}{p}\right) \varepsilon_{p}^{r} \\
=q^{(m-1) / 2} \int_{R_{p}^{\times}} \chi^{-1}(z) d z \sum_{y \in S /\left(1+q R_{p}\right)} \psi_{p}\left(\frac{1}{q}\left(y+\frac{z y^{e}}{2-m}\right)\right) \\
\quad \cdot\left(\frac{c_{2} \cdots c_{r}}{p}\right) \varepsilon_{p}^{r}
\end{aligned}
$$

because $r$ is even, when $a>1$ is odd and $p=p_{j}$ for $j=1, \ldots, s$.

2.3. The case of $a(\chi) \neq a>1$. In this case we have

$$
\int_{R_{p}^{\times}} \chi^{-1}(z) K l_{m}(z ; e ; S) d z=0
$$

because the integral with respect to $x$ on the right side of (1) vanishes. On the other hand the expressions on the right side of (6) and (7) are equal to zero because the integrals there with respect to $x$ vanish. 
2.4. The case of unramified $\chi$. Now we assume that $\chi$ is unramified. Then the right side of (1) vanishes, because $q=p^{a}$ with $a>1$. Therefore (8) holds in this case. By the same reason, the right side of (6) and (7) are zero because the integrals with respect to $z$ vanish.

\section{IDENTITIES OF EXPONENTIAL SUMS}

3.1. Local identities. By the equations in (6) and (7) and results in Subsections 2.3 and 2.4, we prove the following identities of incomplete exponential sums in the case of $a>1$.

Theorem 3. Let $S$ be a subset of $R_{p}^{\times}$satisfying $S\left(1+q R_{p}\right)=S$ where $q=p^{a}$ with $a>1$ and $p$ being an odd prime. Let e be a nonzero integer and $m>1$ an integer congruent to 1 modulo $\phi(q)$, where $\phi$ is the Euler function. Then for $c, p$, and $\psi_{p}$ chosen above we have

$$
\begin{gathered}
\sum_{\substack{b_{1} \in S /\left(1+q R_{p}\right), b_{2}, \ldots, b_{m} \in R_{p}^{\times} /\left(1+q R_{p}\right)}} \psi_{p}\left(\frac{1}{q}\left(b_{1}+\cdots+b_{m}+\frac{z b_{1}^{e}}{b_{2} \cdots b_{m}}\right)\right) \\
=q^{(m-1) / 2} \sum_{y \in S /\left(1+q R_{p}\right)} \psi_{p}\left(\frac{1}{q}\left(y+\frac{z y^{e}}{2-m}\right)\right) \\
=q^{(m-1) / 2}\left(\frac{c_{2} \cdots c_{r}}{p}\right) \varepsilon_{p}^{r} \sum_{\begin{array}{c}
\text { when } a>1\left(1+q R_{p}\right) \\
\text { when } a>1 \text { is even odd }
\end{array}} \psi_{p}\left(\frac{1}{q}\left(y+\frac{z y^{e}}{2-m}\right)\right)
\end{gathered}
$$

for any $z \in R_{p}^{\times}$, where $r$ with $1<r \leq m$ and $c_{2}, \ldots, c_{r}$ with $\left(c_{2}, p\right)=\cdots=$ $\left(c_{r}, p\right)=1$ are solely determined by $p$ and $m$.

3.2. The proof of Theorem 1. These local identities imply Theorem 1. To see this we use the same odd $c=p_{1}^{a_{1}} \cdots p_{s}^{a_{s}}$. For each $j$ let $S_{j}$ be a subset of $R_{p_{j}}^{\times}$such that $S_{j}\left(1+p_{j}^{a_{j}} R_{p_{j}}\right)=S_{j}$. Let $m>1$ be an integer which satisfies $m \equiv 1(\bmod \phi(c))$, where $\phi$ is the Euler function. Fix a nonzero integer $e$. For any integer $z$ relatively prime to $c$ Theorem 3 implies that

$$
\begin{aligned}
& \prod_{1 \leq j \leq s} \sum_{\substack{b_{1 j} \in S_{i} /\left(1+p_{j}^{a_{j}} R_{p_{j}}\right), b_{2 j}, \ldots, b_{m j} \in R_{p_{j}}^{\times} /\left(1+p_{j}^{a_{j}} R_{p_{j}}\right)}} \psi_{p_{j}}\left(\frac{1}{p_{j}^{a_{j}}}\left(b_{1 j}+\cdots+b_{m j}+\frac{z b_{1 j}^{e}}{b_{2 j} \cdots b_{m j}}\right)\right) \\
& =\prod_{1 \leq j \leq s} p_{j}^{a_{j}(m-1) / 2} \sum_{y_{j} \in S_{j} /\left(1+p_{j}^{a_{j}} R_{p_{j}}\right)} \psi_{p_{j}}\left(\frac{1}{p_{j}^{a_{j}}}\left(y_{j}+\frac{z y_{j}^{e}}{2-m}\right)\right) \\
& \prod_{\substack{1 \leq j \leq s, \\
\text { with } a_{j} \text { being odd }}}\left(\frac{c_{2 j} \cdots c_{r_{j} j}}{p_{j}}\right) \varepsilon_{p_{j}}^{r_{j}}
\end{aligned}
$$

where $r_{j}$ and $c_{2 j}, \ldots, c_{r_{j} j}$ are the constants in Theorem 3 for $p=p_{j}, j=1, \ldots, s$. Let $S$ be the subset of a reduced residue system modulo $c$ which consists of numbers $b_{1}$ satisfying $b_{1} \equiv b_{1 j}\left(\bmod p_{j}^{a_{j}}\right)$ for some $b_{1 j} \in S_{j} /\left(1+p_{j}^{a_{j}} R_{p_{j}}\right)$, for all $j=1, \ldots, s$, according to the Chinese remainder theorem. Then the above equality can be 
written as

$$
\begin{aligned}
& \sum_{\substack{\left.b_{1} \in S, b_{2}, \ldots, b_{m} \bmod c, b_{2}, c\right)=\cdots=\left(b_{m}, c\right)=1}} \prod_{1 \leq j \leq s} \psi_{p_{j}}\left(\frac{1}{p_{j}^{a_{j}}}\left(b_{1}+\cdots+b_{m}+z b_{1}^{e} \bar{b}_{2} \cdots \bar{b}_{m}\right)\right) \\
& =c^{(m-1) / 2}\left(\prod_{\substack{1 \leq j \leq s, \\
\text { with } a_{j} \text { odd }}}\left(\frac{c_{2 j} \cdots c_{r_{j} j}}{p_{j}}\right) \varepsilon_{p_{j}}^{r_{j}}\right) \sum_{y \in S} \prod_{1 \leq j \leq s} \psi_{p_{j}}\left(\frac{y+z(\overline{2-m}) y^{e}}{p_{j}^{a_{j}}}\right) .
\end{aligned}
$$

We rewrite the above in terms of $\bar{\varphi}_{p_{j}}$. Note that $\bar{\varphi}_{p}(x / c)=1$ for any integer $x$, if $p \neq p_{1}, \ldots, p_{s}$. Since the global character $\varphi$ is trivial on $\mathbb{Q}$, we can replace the product of $\bar{\varphi}_{p_{j}}$ by $\varphi_{\mathbb{R}}$ and the equation above becomes

$$
\begin{aligned}
& \sum_{\substack{b_{1} \in S, b_{2}, \ldots, b_{m} \bmod c,\left(b_{2}, c\right)=\cdots=\left(b_{m}, c\right)=1}} \mathrm{e}\left(\frac{1}{c}\left(b_{1}+\cdots+b_{m}+z b_{1}^{e} \bar{b}_{2} \cdots \bar{b}_{m}\right)\right) \\
& =c^{(m-1) / 2}\left(\prod_{\substack{1 \leq j \leq s, \\
\text { with } a_{j} \text { odd }}}\left(\frac{c_{2 j} \cdots c_{r_{j} j}}{p_{j}}\right) \varepsilon_{p_{j}}^{r_{j}}\right) \sum_{y \in S} \mathrm{e}\left(\frac{1}{c}\left(y+z(\overline{2-m}) y^{e}\right)\right) .
\end{aligned}
$$

This completes the proof of Theorem 1.

Q.E.D.

\section{ACKNOWLEDGEMENT}

The author would like to express thanks to Wenzhi Luo for helpful discussions.

\section{REFERENCES}

[1] D. Bump, W. Duke, J. Hoffstein, and H. Iwaniec, An estimate for the Hecke eigenvalues of Maass forms, Inter. Math. Res. Notices, 4 (1992), 75-81. MR 93d:11047

[2] H. Davenport and H. Hasse, Die Nullstellen der Kongruenzzetafunktionen in gewissen zyklischen Fällen, J. reine angew. Math., 172 (1935), 151-182.

[3] H. Jacquet and Y. Ye, Relative Kloosterman integrals for GL(3), Bulletin de la Société mathématique de France, 120 (1992), 263-295. MR 94c:11047

[4] W. Luo, Bounds for incomplete hyper-Kloosterman sums, preprint.

[5] W. Luo, Z. Rudnick, and P. Sarnak, On Selberg's eigenvalue conjecture, Geom. Funct. Anal., 5 (1995), 387-401. MR 96h:11045

[6] Z. Mao and S. Rallis, A trace formula for dual pairs, Duke Math. J., 87 (1997), 321-341. CMP 97:11

[7] Y. Ye, Kloosterman integrals and base change for $G L(2)$, J. reine angew. Math. 400 (1989), 57-121. MR 90i:11134

[8] Y. Ye, The lifting of Kloosterman sums, J. Number Theory, 51 (1995), 275-287. MR 97a: 11126

[9] Y. Ye, The lifting of an exponential sum to a cyclic algebraic number field of a prime degree, Transactions Amer. Math. Soc. 350 (1998), 5003-5015. CMP 97:08

[10] Y. Ye, Exponential sums for $G L(n)$ and their applications to base change, J. Number Theory 68 (1998), 112-130. CMP 98:07

[11] D. Zagier, Modular forms associated to real quadratic fields, Invent. Math. 30 (1975), 1-46. MR 52:3062

Department of Mathematics, The University of Iowa, Iowa City, Iowa 52242-1419

E-mail address: yey@math.uiowa.edu

Current address: Department of Mathematics, The University of Hong Kong, Hong Kong 